\title{
Utilidad de un sistema de registro de una derivación electrocardiográfica y transmisión inalámbrica durante la pandemia por COVID-19
}

\author{
Electrocardiographic Recording System and Wireless Transmission During the COVID-19 \\ Pandemic

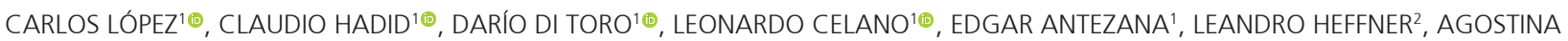 \\ BRUNO $^{2}$, CLARISA GASHU ${ }^{2}$, MARCELO ZYLBERMAN², CARLOS LABADET ${ }^{1 \oplus}$
}

\section{RESUMEN}

Introducción: Se ha comunicado que algunos tratamientos utilizados para la infección por COVID-19 pueden ocasionar alteraciones del intervalo QT y arritmias graves. La medición por electrocardiograma (ECG) convencional requiere personal adicional y riesgo de contagio. Nuevas tecnologías para obtención de un ECG conectados a teléfonos inteligentes (smartphones) proporcionan una alternativa para evaluación del QTc.

Objetivo: El objetivo fue evaluar la factibilidad de un dispositivo para registro electrocardiográfico de un canal, para la medición del intervalo QT en pacientes con sospecha o confirmación de infección por COVID-19, antes de recibir drogas que prolongan el intervalo QT.

Material y métodos: Se obtuvieron registros de ECG con un dispositivo Kardia Mobile (KM) con trasmisión a un smarthphone. La sección de electrofisiología cardíaca centralizó la recepción por medio electrónico de los ECG en formato de archivo pdf y realizó las mediciones de los intervalos QTm y QTc.

Resultados: Se estudiaron 31 pacientes, edad promedio 61 años (rango 20-95 años), sospechosos de presentar infección por COVID-19 enrolados para tratamiento con hidroxicloroquina, azitromicina, ritonavir y lopinavir. Los registros pudieron ser leídos en todos los casos, y debieron repetirse en dos casos. Los valores del intervalo QTc promedio en varones y mujeres fue $423 \mathrm{mseg}$ (rango 380-457 mseg) y $439 \mathrm{mseg}$ (rango 391-540 mseg), respectivamente. El tiempo de respuesta desde el envío del ECG al grupo de análisis fue 11 min (rango 1-155).

Conclusiones: Los registros ECG obtenidos con dispositivos KM, para trasmisión a un smartphone a un grupo central de lectura, permitieron la medición del intervalo QTe en todos los pacientes.

Palabras claves: Infección por Coronavirus - COVID-19 - Arritmias cardíacas - Teléfono Inteligente - Aplicaciones Móviles - Electrocardiografía Ambulatoria/ instrumentación

\begin{abstract}
Background: Some therapies used for COVID-19 can prolong the QT interval and produce severe arrhythmias. QT interval measured from a standard electrocardiogram (ECG) requires additional personnel and risk of infection. Novel technologies to obtain an ECG connected to smartphones provide an alternative for the evaluation of corrected QT interval (QTc). Objective: The aim of this study was to evaluate the feasibility of using a single-lead ECG device to measure the QT interval in patients with suspected or confirmed COVID-19 before receiving treatment with drugs that can prolong the QT interval. Methods: The ECG was obtained with a KardiaMobile (KM) device and transmitted to a smartphone. The ECG recordings were saved as pdf files and electronically submitted to the electrophysiology section which centralized the reception and assessed the measured QT and QTc intervals.

Results: A total of 31 patients (mean age 61 years, range 20-95 years) with suspected COVID-19 enrolled for treatment with hydroxychloroquine, azithromycin, ritonavir or lopinavir were analyzed. The recordings could be read in all the cases and had to be repeated in two cases. The mean value of the QTc interval was $423 \mathrm{~ms}$ (range 380-457 ms) in men and $439 \mathrm{~ms}$ (range 391-540 ms) in women. The response time since the ECG recording was submitted for analysis was 11 min (range 1-155). Conclusions: The QTc interval could be measured from ECG recordings obtained with KM devices connected to a smartphone and transmitted to a centralized reading center in all patients.
\end{abstract}

Key words: Coronavirus Infections - COVID-19 - Arrhythmias, Cardiac - Smartphone - Mobile Applications - Electrocardiography, Ambulatory/instrumentation

Rev Argent CARDiol 2020;88;211-215. http://dx.doi.org/10.7775/rac.es.v88.i3.18000

Dirección para separatas: Carlos Labadet - Pi y Margall 750 - C1155 AHD, Buenos Aires, Argentina. E-mail: clabadet@gmail.com Tel: +54-11-4121-0700 (int. 2821)

\footnotetext{
${ }^{1}$ Sección Electrofisiología, Hospital Gral. de Agudos Dr. Cosme Argerich, Buenos Aires, Argentina.

${ }^{2}$ División Cardiología, División Clínica Médica, Hospital Gral. de Agudos Dr. Cosme Argerich, Buenos Aires, Argentina.
} 


Abreviaturas
\begin{tabular}{ll|l} 
ptes. Pacientes & QTC QT corregido \\
SP $\quad$ Teléfonos celulares inteligentes & EPP Equipos de protección personal \\
NICE Instituto Nacional para la Excelencia en la Atención Médica &
\end{tabular}

\section{INTRODUCCIÓN}

Actualmente algunos de los tratamientos implementados en la comunidad médica para el tratamiento de la neumonía por COVID-19, incluyen fármacos como la hidroxicloroquina, azitromicina, ritonavir o lopinavir. Estos fármacos pueden estar asociados a la prolongación del intervalo QT del ECG y la potencial aparición de arritmias ventriculares malignas, por lo cual se sugiere un control estricto de estos efectos adversos durante el tratamiento. $(1,2)$

En una situación compleja como es el desborde sanitario por una pandemia, la realización de un ECG de 12 derivaciones se convierte en un acto engorroso, debido a la participación de personal dedicado, sumado a la movilidad y la desinfección de un electrocardiógrafo con sus conexiones. La propiedad del virus de sobrevivir en fómites por varias horas o días es uno de los elementos que hacen que la enfermedad tenga una alta transmisibilidad. Con la finalidad de proteger al personal del sistema de salud, minimizar los riesgos y reducir la carga laboral de los médicos que están en primera línea de atención, desde el área de electrofisiología hemos propuesto la utilización de dispositivos portátiles para el registro de una derivación electrocardiográfica.

\section{OBJETIVOS}

El objetivo de este estudio fue evaluar la factibilidad en la utilización de un dispositivo para registro electrocardiográfico de una derivación, con conexión a distancia a un teléfono inteligente, para la medición centralizada del intervalo QT en pacientes (ptes.) con sospecha o confirmación de infección por COVID-19, antes de recibir tratamiento con drogas que pueden prolongar el intervalo QT.

\section{MATERIAL Y MÉTODOS}

Se utilizó un dispositivo portátil para registro de una derivación electrocardiográfica de contacto simple KM (AliveCor Inc., San Francisco, CA, EE. UU.). El mismo consiste en un pequeño dispositivo, con un peso de $18 \mathrm{~g}$, de pequeñas dimensiones $(8,2 \mathrm{~cm} \times 3,2 \mathrm{~cm} \times 0,35 \mathrm{~cm})$, portátil, capacidad de registro de una señal de ECG y disponible para conectarse vía Bluetooth con teléfonos celulares inteligentes (SP) con plataforma iOS y Android. El dispositivo está constituido por dos placas conductoras (dos electrodos de acero inoxidable de $3 \mathrm{~cm} \times 3 \mathrm{~cm}$ ) para contacto con los dedos del paciente y registrar una derivación bipolar (Figura 1). El dispositivo cuenta con la aprobación del Instituto Nacional para la Excelencia en la Atención Médica (NICE) y ha mostrado su utilidad en la valoración de intervalos del ECG en situaciones particulares como el efecto de fármacos que prolongan el QT como el Dofetilide. $(3,4)$

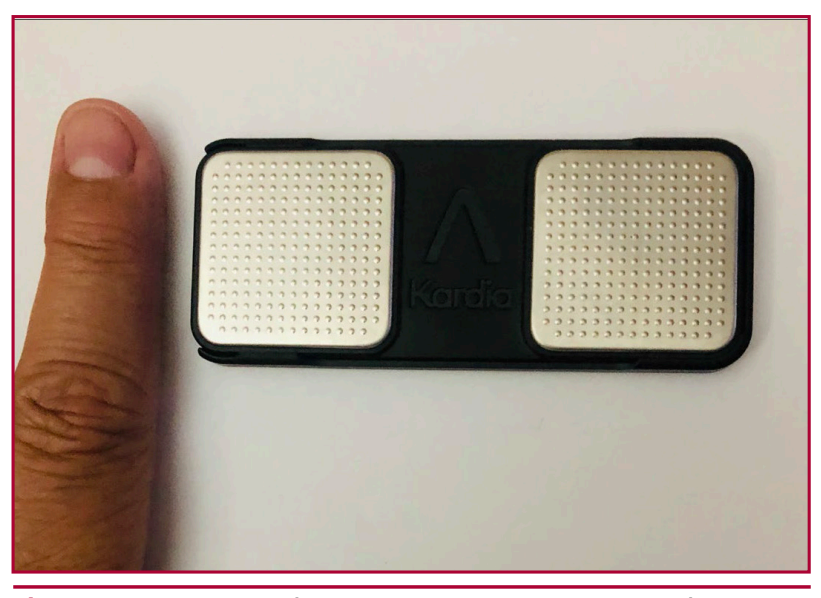

Fig. 1. Dispositivo portátil para registro de una derivación electrocardiográfica de contacto simple Kardia Mobile (AliveCor Inc., San Francisco, CA, EE.UU.)

El grupo de estudio consistió en pacientes consecutivos incluidos de manera prospectiva a nuestro hospital por neumopatía y sospecha o confirmación de infección por COVID-19 antes del inicio de tratamiento con hidroxicloroquina y azitromicina. Todos los profesionales de la salud no familiarizados con el manejo del sistema fueron entrenados en el manejo de KM. Todos los registros electrocardiográficos de ingreso fueron tomados y almacenados en teléfono celular personal de cada médico coordinador utilizando la herramienta específica compatible con sistemas iOS o Android, posteriormente enviados en formato PDF al grupo de electrofisiología cardíaca del hospital en forma remota para definir los valores del intervalo QT corregido (QTc). Se aplicó un algoritmo creado específicamente para este fin, considerando las mediciones basales del QTc del registro KM y se determinó el score de Tisdale para conocer el riesgo individual de prolongación del QT durante la internación. (5) Se utilizó un programa específico para la medición digital (EP Calipers). La fórmula de Bazett fue la seleccionada para la medición del intervalo QTc. En los casos con QRS con duración mayor de 120 milisegundos (ms) se estableció la fórmula de Bogossian con reducción del 50\% de la duración del QRS al valor hallado con la fórmula de Bazett. (6) Correlacionamos el QTc medido por Kardia y por un ECG convencional en un grupo control de 12 ptes. internados por otras patologías, cuyas mediciones se realizaron por dos investigadores en forma ciega.

\section{Análisis estadístico}

Para describir los datos se utilizó medias y desviación estándar o mediana y rango de acuerdo a su distribución. En el grupo control, se realizó el análisis de regresión lineal calculando el coeficiente de correlación de Pearson. Se utilizó IBM SPSS Statistics para Macintosh 20.0 (Armonk, NY, Estados Unidos: IBM Corp.).

\section{Consideraciones éticas}

Se informó al jefe del servicio de cardiología el interés de la sección por llevar a cabo este estudio y se solicitó su 
autorización, este se realizó siguiendo las guías de buenas prácticas clínicas y las normativas legales vigentes.

\section{RESULTADOS}

Sobre el grupo control de 12 ptes. se midió el intervalo QTc utilizando ECG de 12 derivaciones (426 $\pm 43 \mathrm{~ms})$ y KM (434 $\pm 52 \mathrm{~ms}$ ) (Figura 2). Se observó una muy buena correlación entre ambas mediciones (coeficiente $\mathrm{r}^{2}$ 0,905, Figura 3).

Entre el 2 de abril de 2020 y el 9 de mayo de 2020 se recibieron 31 registros, 12 de ptes. femeninas y 19 de masculinos, edad promedio 61 años (rango 20-95 años). Los registros obtenidos fueron óptimos y permitieron la medición del intervalo QT y determinar el QTc en todos los ptes. En dos ptes., se aconsejó la obtención de un nuevo registro alternativo, ubicando el $\mathrm{KM}$ al nivel precordial, a la altura de la derivación V1-V2 para confirmar la medición previa (Figura 4). El ECG presentó ritmo sinusal en 29 ptes. con un intervalo PR promedio de $144 \mathrm{~ms}$ (rango 120-180 ms), uno presentó fibrilación auricular y otro, ritmo de la unión AV al ingreso. Veinticinco ptes. presentaron complejo QRS angosto (duración de QRS $90 \mathrm{~ms}$ [rango 80-120 ms], seis ptes. presentaron un complejo QRS ensanchado con un promedio de 153,3 ms (rango 140-160 ms). La media de la frecuencia cardíaca fue de 90,8 latidos por minuto. El intervalo QTc fue normal para recibir
Fig. 2. A) Medición de un intervalo QTc prolongado en la derivación $D$ I en un registro electrocardiográfico de superficie. B) Medición mediante registro Kardia Mobile del intervalo QT en el mismo paciente.
Fig. 3. Correlación entre QTc medido por ECG (QTCE) y QTc medido por Kardia Mobile (QTcK). Scatter plot.
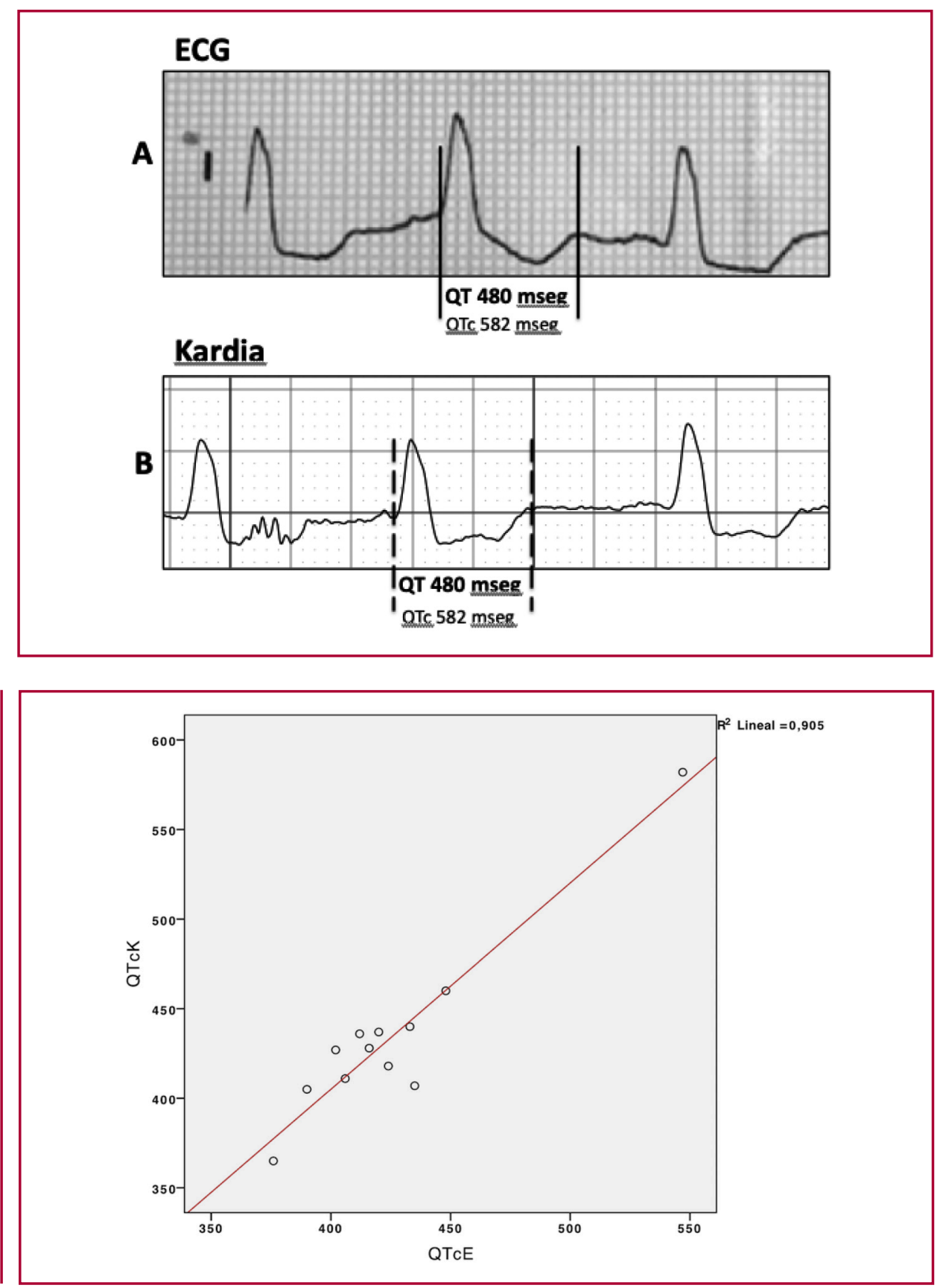

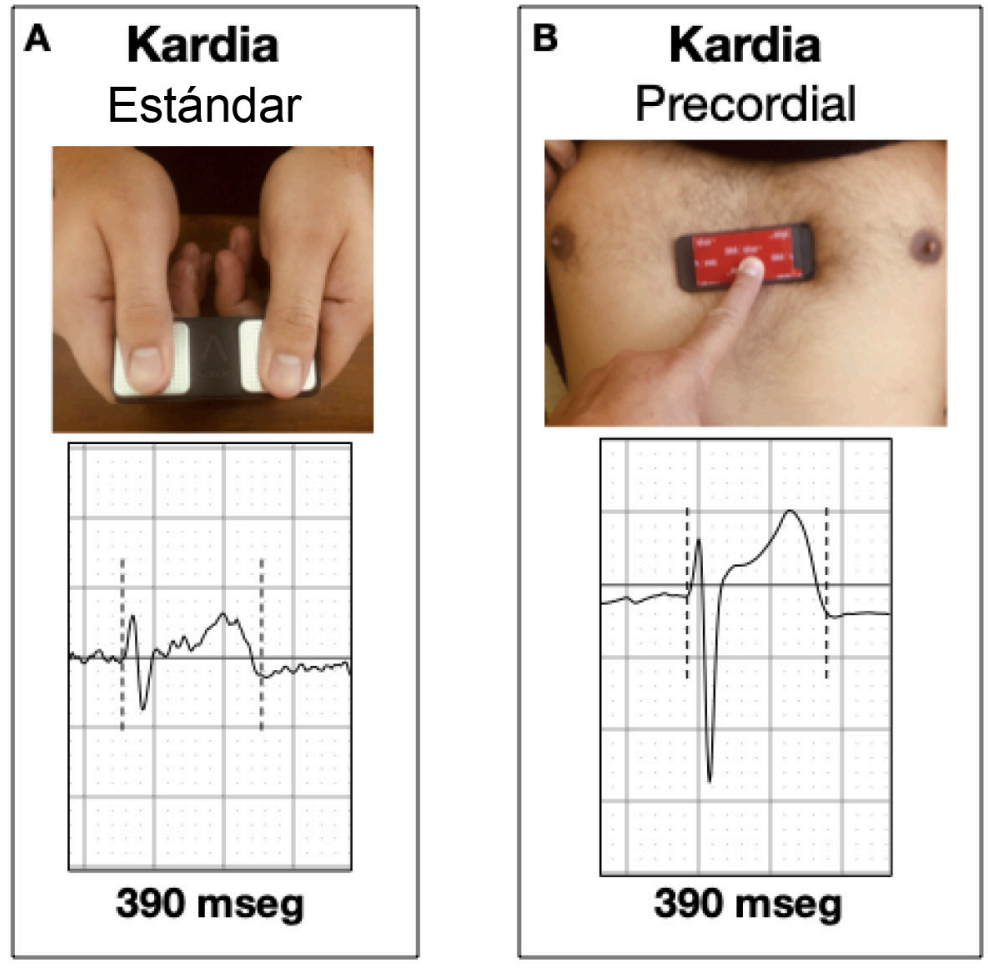

Fig. 4. A) Registro estándar tomado con un dispositivo Kardia Movile. B) Registro en el mismo paciente con un dispositivo Kardia Mobile en posición V1-V2 para valoración del intervalo QT.

y mantener tratamiento en 30 ptes., solo en un caso se aconsejó valorar riesgo/beneficio del tratamiento por presentar intervalo QTc prolongado de $540 \mathrm{~ms}$. Finalmente, no se inició tratamiento farmacológico en ese paciente. Los valores obtenidos del intervalo QTc promedio en varones fue de $423 \mathrm{~ms}$ (rango 380-457 $\mathrm{ms}$ ) y en las mujeres fue en promedio de $439 \mathrm{~ms}$ (rango 391-540 ms). El tiempo de respuesta con la medición, desde el momento del envío del registro al grupo de análisis QT fue de 11 min (mediana).

\section{DISCUSIÓN}

Los hallazgos de nuestro estudio muestran que la medición del intervalo QTc a través de un dispositivo KM es factible y evita la participación de personal adicional de salud y equipamiento suplementario con los beneficios lógicos en una enfermedad de alta contagiosidad.

La ventaja del sistema KM es su simplicidad, por lo que puede ser utilizado por el mismo personal que asiste diariamente al paciente, como la toma de un signo vital más, con lo que se evita la participación de personal adicional. En esta experiencia, también pudimos observar que la lectura centralizada puede colaborar con los médicos que se encuentran en primera línea de atención de pacientes con COVID-19.

La obtención de un registro electrocardiográfico para realizar mediciones del intervalo QT requiere de personal técnicamente entrenado y especializado más un equipamiento adicional con una importante inver- sión de tiempo en la obtención y en la desinfección de dicho equipo. El KM es muy sencillo de desinfectar por su pequeño tamaño y ausencia de cables. También es conocido que existen recomendaciones para la medición del intervalo QT y menciones que subestiman la medición del intervalo QT utilizando una sola derivación del ECG. (7) Otras publicaciones han mencionado la utilización de nuevas tecnologías para medición del intervalo QT tanto en la población pediátrica como en la edad adulta, que emplean una sola derivación con resultados fiables y contundentes en cuanto a su utilidad. $(8,9)$ No obstante, en nuestro grupo control obtuvimos muy buena correlación entre el QTc obtenido con KM y el ECG convencional.

Algunos registros requirieron la obtención de una muestra adicional alternativa para confirmar las mediciones obtenidas de manera estándar; se optó de manera original por un registro precordial bipolar a la altura de las derivaciones V1-V2. Esta modalidad ha sido descripta como otra alternativa de registro para situaciones especiales. (10)

Algunas comunicaciones han hecho referencia a la presencia de ruido en la línea de base del registro obtenido con la tecnología KM. (11) Esta dificultad fue una de las limitaciones en nuestro estudio. El porcentaje de registros con algún tipo de ruido inconveniente en la línea de base se observó en 7 ptes. (21\%), pero solo en 2 ptes. (6\%) de los registros obtenidos con el dispositivo $\mathrm{KM}$ fue un inconveniente para el equipo de especialistas 
en la medición del intervalo QT y se solicitó un nuevo registro. Esta dificultad puede superarse si se repite el registro o, si se opta por la derivación precordial, se pueden obtener registros limpios que permiten la realización de una medición apropiada. En una reciente comunicación, el American College of Cardiology recomendó que para reducir al máximo la exposición al COVID-19 o ahorrar recursos de Equipos de Protección Personal (EPP), el monitoreo del intervalo QTc se puede realizar utilizando sustitutos para la evaluación de ECG de 12 derivaciones, como los dispositivos móviles del tipo KM. (12)

\section{Limitaciones:}

Se trata de una experiencia inicial en un hospital universitario, de derivación del sistema público de salud. Estos resultados pueden no ser extrapolables a centros periféricos.

El dispositivo KM utilizado registró trazados de una sola derivación. Si bien existen comunicaciones acerca de su confiabilidad para la medición del QT, no puede soslayarse el hecho de que los trazados de 6 o 12 derivaciones proveen una información más completa del tiempo de repolarización ventricular, sobre todo si incluyen derivaciones precordiales.

En nuestra experiencia, un 21\% de los trazados presentaron algún tipo de ruido, pero solo en 2 ptes. (6\%) el registro debió repetirse y, con ello, el intervalo QT pudo medirse en todos los ptes.

\section{CONCLUSIONES}

La obtención de un registro de un canal electrocardiográfico para valoración del intervalo QTc con un dispositivo KM que pueda ser analizado en forma digital, es factible en condiciones de pandemia. Los casos de registro con ruido pueden solucionarse con la repetición de la toma o recurriendo a una derivación precordial bipolar alternativa.

\section{BIBLIOGRAFÍA}

1. Mercuro NJ, Yen CF, Shim DJ, Maher TR, McCoy CM, Zimetbaum PJ, et al. Risk of QT Interval Prolongation Associated With Use of Hydroxychloroquine With or Without Concomitant Azithromycin Among Hospitalized Patients Testing Positive for Coronavirus Disease
2019 (COVID-19). JAMA Cardiol 2020; May 1, e201834 http://doi. org/10.1001/jamacardio.2020.1834

2. Bessière, F., Roccia, H., Delinière, A., Charrière, R., Chevalier, P., Argaud, L., \& Cour, M. (2020). Assessment of QT Intervals in a Case Series of Patients With Coronavirus Disease 2019 (COVID-19) Infection Treated With Hydroxychloroquine Alone or in Combination With Azithromycin in an Intensive Care Unit. JAMA Cardiol 2020; e201787.http://doi.org/10.1001/jamacardio.2020.178.

3. National Institute for Clinical Excellence (NICE). AliveCor Heart Monitor and AliveECG app (Kardia Mobile) for Detecting Atrial Fibrillation 2015. Available online: $\mathrm{https://www.nice.org.uk/advice/mib35.}$ 4. Muhlestein JB. QTC intervals can be assessed with the AliveCor heart monitor in patients on dofetilide for atrial fibrillation. J Electrocardiol 2015;48:10-1. http://doi.org/10.1016/j.jelectrocard.2014.11.007 5. Tisdale JE, Jayes HA, Kingery JR, Mourad NA, Trujillo TN, Overholser BR, et al. Development and validation of a risk score to predict QT interval prolongation in hospitalized patients. Circ Cardiovasc Qual Outcomes 2013;6:479-87. http://doi.org/10.1161/ CIRCOUTCOMES.113.000152

6. Bazett HC. An analysis of the time-relations of electrocardiograms. Heart. 1920;7:353-70.

7. Kligfield P, Tyl B, Maarek M, Maison-Blanche P. Magnitude, mechanism, and reproducibility of QT interval differences between superimposed global and individual lead ECG complexes. Ann Noninvas Electrocardiol. 2007;12:145-52.

8. Kligfield P, Tyl B, Maarek M, Maison-Blanche P. Magnitude, mechanism, and reproducibility of QT interval differences between superimposed global and individual lead ECG complexes. Ann Noninvas Electrocardiol 2007;12:145-52. http://doi.org/10.1111/j.1542474X.2007.00153.x

9. Karacan M, Celik N, Gul EE, Akdeniz C, Tuzcu V.Validation of a smartphone-based electrocardiography in the screening of QT intervals in children. North Clin Istanb 2019;6:48-52. http://doi. org/10.14744/nci.2018.44452

10. Maron BJ, Friedman RA, Kligfield P, Levine BD, Viskin S, Chaitman BR, et al. Assessment of the 12-Lead ECG as a Screening Test for Detection of Cardiovascular Disease in Healthy General Populations of Young People (12-25 Years of Age). Circulation 2014;130:1303-34. http://doi.org/10.1161/CIR.0000000000000025

11. Mc Loughlin MJ. Precordial bipolar leads: A new method to study anterior acute myocardial infarction. J Electrocardiol 2020; 59:45-64. http://doi.org/10.1016/j.jelectrocard.2019.12.017

12. Marston HR, Hadley R, Banks D, Duro MDCM. Mobile SelfMonitoring ECG Devices to Diagnose Arrhythmia that Coincide with Palpitations: A Scoping Review. Healthcare (Basel) 2019 7:96. http:// doi.org/10.3390/healthcare7030096

13. Simpson TF, Kovacs RJ, Stecker EC. Ventricular Arrhythmia Risk Due to Hydroxychloroquine-Azithromycin Treatment For COVID-19. Cardiology Magazine 2020;Mar 29. Disponible en: https://www. acc.org/latest-in-cardiology/articles/2020/03/27/14/00/ventriculararrhythmia-risk-due-to-hydroxychloroquine-azithromycin-treatmentfor-covid-19. 